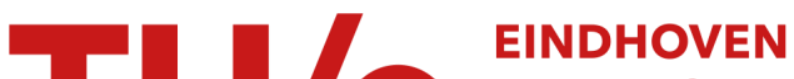 \\ UNIVERSITY OF \\ TECHNOLOGY
}

\section{Voltage-gated pinning in a magnetic domain-wall conduit}

\section{Citation for published version (APA):}

Franken, J. H., Yin, Y., Schellekens, A. J., Brink, van den, A., Swagten, H. J. M., \& Koopmans, B. (2014). Voltage-gated pinning in a magnetic domain-wall conduit. Applied Physics Letters, 103(10), 102411-1/5.

[102411]. https://doi.org/10.1063/1.4819771

DOI:

10.1063/1.4819771

Document status and date:

Published: 01/01/2014

\section{Document Version:}

Publisher's PDF, also known as Version of Record (includes final page, issue and volume numbers)

\section{Please check the document version of this publication:}

- A submitted manuscript is the version of the article upon submission and before peer-review. There can be important differences between the submitted version and the official published version of record. People interested in the research are advised to contact the author for the final version of the publication, or visit the $\mathrm{DOI}$ to the publisher's website.

- The final author version and the galley proof are versions of the publication after peer review.

- The final published version features the final layout of the paper including the volume, issue and page numbers.

Link to publication

\section{General rights}

Copyright and moral rights for the publications made accessible in the public portal are retained by the authors and/or other copyright owners and it is a condition of accessing publications that users recognise and abide by the legal requirements associated with these rights.

- Users may download and print one copy of any publication from the public portal for the purpose of private study or research.

- You may not further distribute the material or use it for any profit-making activity or commercial gain

- You may freely distribute the URL identifying the publication in the public portal.

If the publication is distributed under the terms of Article 25fa of the Dutch Copyright Act, indicated by the "Taverne" license above, please follow below link for the End User Agreement:

www.tue.nl/taverne

Take down policy

If you believe that this document breaches copyright please contact us at:

openaccess@tue.nl

providing details and we will investigate your claim. 


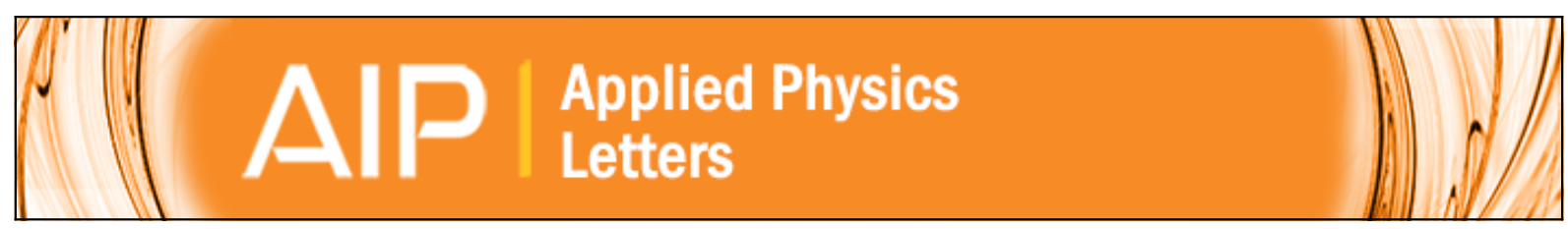

\section{Voltage-gated pinning in a magnetic domain-wall conduit}

J. H. Franken, Y. Yin, A. J. Schellekens, A. van den Brink, H. J. M. Swagten, and B. Koopmans

Citation: Applied Physics Letters 103, 102411 (2013); doi: 10.1063/1.4819771

View online: http://dx.doi.org/10.1063/1.4819771

View Table of Contents: http://scitation.aip.org/content/aip/journal/apl/103/10?ver=pdfcov

Published by the AIP Publishing

\section{Over 700 papers \& presentations on}

multiphysics simulation vew now

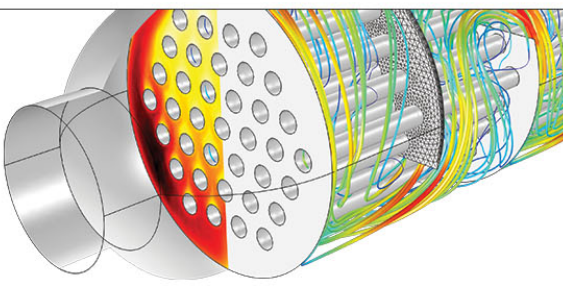




\title{
Voltage-gated pinning in a magnetic domain-wall conduit
}

\author{
J. H. Franken, ${ }^{\text {a) }}$ Y. Yin, A. J. Schellekens, A. van den Brink, H. J. M. Swagten, \\ and B. Koopmans \\ Department of Applied Physics, Center for NanoMaterials, Eindhoven University of Technology, \\ P.O. Box 513, 5600 MB Eindhoven, The Netherlands
}

(Received 8 July 2013; accepted 16 August 2013; published online 6 September 2013)

\begin{abstract}
In spintronic devices relying on magnetic domain-wall (DW) motion, robust control over the DW position is required. We use electric-field control of perpendicular magnetic anisotropy to create a voltage-gated pinning site in a microstructured $\mathrm{Pt} / \mathrm{Co} / \mathrm{AlO}_{x} \mathrm{DW}$ conduit. A DW pins at the edge of a gate electrode, and the strength of pinning can be tuned linearly and reversibly with an efficiency of $0.22(1) \mathrm{mT} / \mathrm{V}$. This result is supported by a micromagnetic model, taking full account of the anisotropy step at the gate edge, which is directly caused by a change in the electron density due to the choice of material. @ 2013 AIP Publishing LLC. [http://dx.doi.org/10.1063/1.4819771]
\end{abstract}

In recent years, control of ferromagnetic (FM) devices by electric fields has received a lot of attention due to very promising prospects for low-power memory and logic devices. In particular, it was found that applying a voltage to a thin magnetic film across a gate dielectric can reversibly alter the magnetic properties, such as the magnetic anisotropy, ${ }^{1-8}$ coercivity, ${ }^{9,10}$ saturation magnetization, ${ }^{1,11,12}$ and Curie temperature. ${ }^{11,13}$ This has been demonstrated to be of use in exciting applications such as low-dissipative voltage-driven switching, ${ }^{14,15}$ voltage-gated switching by the Spin Hall effect, ${ }^{16}$ controlling the domain structure, ${ }^{17-19}$ and control over magnetic domain-wall (DW) velocities. ${ }^{20-24}$ Several mechanisms can be responsible for these effects: strain transfer from a ferroelectric layer, ${ }^{17-19}$ changes to the occupation of electron orbitals at the FM/oxide interface, ${ }^{1}$ charge trapping, ${ }^{25}$ and finally, migration of $\mathrm{O}$ ions from the interface. ${ }^{26}$

Recently, Bauer et $a l .{ }^{26}$ have been able to create pinning sites in a DW conduit which can be reprogrammed by applying a voltage. This is particularly promising for application in current-driven DW racetrack memory, ${ }^{27}$ in which a long train of magnetic DWs propagates through a nanowire which functions as a DW conduit. Reproducible operation of such a device requires well-defined pinning sites, which are typically realized by locally altering the geometry ${ }^{28}$ or material properties. ${ }^{29-31}$ The ability to activate and deactivate these pinning sites, preferably at fast timescales, provides new device options and could greatly reduce the current density needed to move the domain walls from site to site. The devices by Bauer $e t$ al. ${ }^{26}$ relied on the effect of electromigration, which has its own advantages and disadvantages. BernandMantel $e$ e al $^{24}$ also showed DW pinning at the boundary of a large electrode by the completely different effect of interface charging, although a systematic analysis of the tunability of the effect was still lacking.

In this letter, we have equipped a microstructured DW conduit with a voltage-gated pinning site which functions by changing the electron density at the FM/oxide interface. The effect is demonstrated in $\mathrm{Pt} / \mathrm{Co} / \mathrm{AlO}_{x}$, a material that is also

\footnotetext{
${ }^{\text {a) }}$ Author to whom correspondence should be addressed. Electronic mail: j.h.franken@tue.nl. Telephone: +31-40-247-4305.
}

very interesting for device applications since it exhibits very fast current-induced DW motion. ${ }^{32}$ We show that the pinning strength experienced by a DW at the edge of a gate electrode is enhanced or decreased reversibly and linearly by a positive or negative voltage. The linear relation between pinning strength and voltage is supported by a micromagnetic model, taking into account the details of the electric field distribution that create the step in the anisotropy leading to pinning.

Devices were fabricated following the design of Figure 1(a). The devices consist of a bottom Pt $(4 \mathrm{~nm}) / \mathrm{Co}(0.8 \mathrm{~nm})$ and a top Pt $(20 \mathrm{~nm})$ electrode, separated by a $10 \mathrm{~nm} \mathrm{AlO}_{x}$ insulating layer. The bottom $\mathrm{Pt} / \mathrm{Co} / \mathrm{AlO}_{x}$ electrode shows perpendicular magnetic anisotropy (PMA) ${ }^{33}$ and is fabricated by means of Electron Beam Lithography (EBL) followed by sputtering of $\mathrm{Pt} / \mathrm{Co} / \mathrm{Al}$, plasma oxidation of the $\mathrm{Al}$ layer, and lift-off. It is shaped to function as a conduit for magnetic DWs by patterning it into a $1 \mu \mathrm{m}$ wide wire with a large contact pad attached to it on one end, while the other end is tapered. After fabrication of the bottom electrode, an additional $\mathrm{AlO}_{x}$ layer is deposited everywhere to ensure gate insulation, and the sample is annealed at $300^{\circ} \mathrm{C}$. After that, the top Pt electrode is fabricated by EBL.

The I-V characteristic of the fabricated device is shown in Figure 1(b). It is seen that the $\mathrm{AlO}_{x}$ layer functions as a tunnel barrier, with a tunneling current that is negligibly small between $-4 \mathrm{~V}$ and $+4 \mathrm{~V}$, allowing us to exclude current-induced effects on the magnetization. In our main experiment, we therefore apply voltages in this range and study how DW pinning at the electrode position is affected. The idea of the experiment is as follows (see Figure 1(c)): by applying a voltage of the appropriate sign, the anisotropy in the DW conduit is increased in the gate region. A perpendicular magnetic field $H$ is applied to move a DW from left to right through the conduit. The DW experiences a step $\Delta K$ in the anisotropy before entering the gate region (Figure 1(d)). Since the energy cost of a DW increases with $\sqrt{K}$, this creates a step in the DW energy landscape at the edge of the gate, where the DW tends to be pinned (Figure 1(e)). The pinning can be overcome by applying a stronger $H$-field, which tilts the energy landscape due to the added Zeeman contribution (Figure 1(f)). 

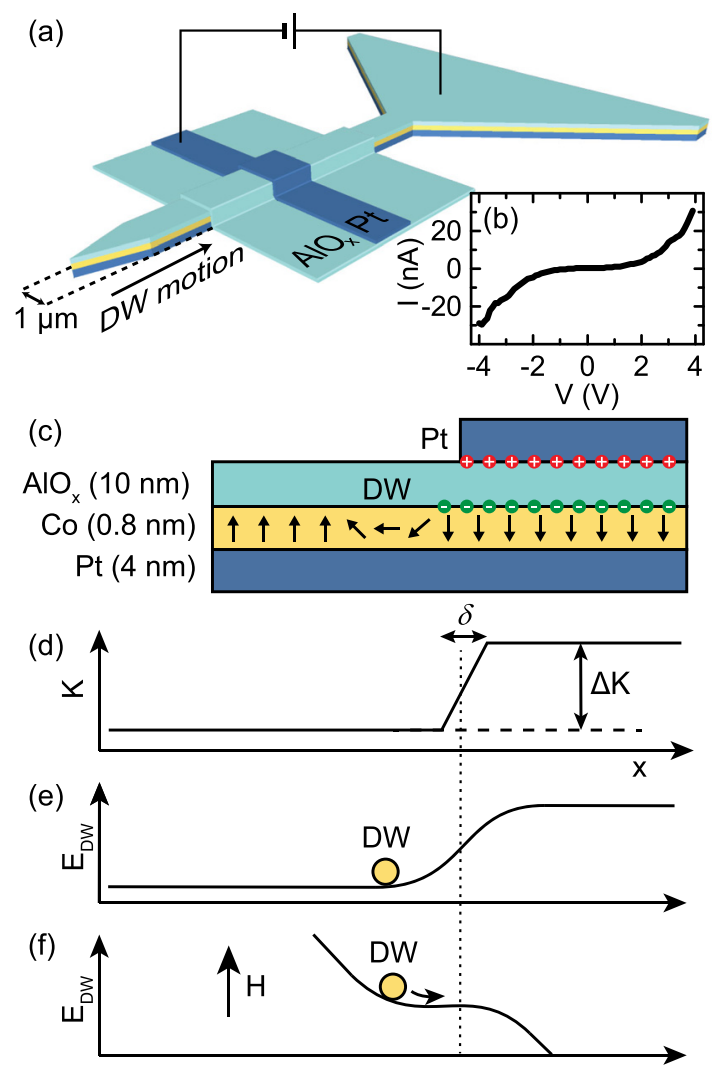

FIG. 1. (a) Sketch of the fabricated device; (b) I-V characteristic of the device; (c) sketch of the situation in the gate region: the excess electrons at the $\mathrm{AlO}_{x} / \mathrm{Co}$ interface enhance the magnetic anisotropy, thereby creating a step in the anisotropy at the gate boundary as sketched in (d). This leads to a DW being pinned in the DW energy landscape sketched in (e). Upon application of a magnetic field $H$ in (f), the DW can be depinned.

The motion of DWs through the fabricated DW conduit is observed directly using Kerr microscopy (Figure 2(a)). First, we describe the situation without an applied voltage (middle row of images). Starting from negative saturation, the external magnetic field is increased to $4 \mathrm{mT}$ and a DW is nucleated. Interestingly, the DW does not nucleate in the contact pad as intended, but rather at the tapered end, an effect that was recently described in detail. ${ }^{34}$ After nucleation, the DW propagates towards the gate. It is observed that the DW stops to the left of the gate (left image), even when the voltage is zero. Since this is observed in almost all fabricated devices, we believe the material properties are slightly different underneath the contact due to, i.e., strain effects or fabrication details, which create a pinning position for the DWs. However, the pinning strength is quite weak and of the same order as natural pinning sites which occur randomly in $\mathrm{Pt} / \mathrm{Co} /$ $\mathrm{AlO}_{x}$ DW conduits. The DWs can therefore still be moved past the gate by applying a slightly higher z-field of $5.0 \mathrm{mT}$ (see the change between the left and center images at $0 \mathrm{~V}$ ); this field is referred to as the pinning field. We now repeat the experiment for various applied voltages and study how the pinning strength is altered. Kerr microscopy snapshots for $-4 \mathrm{~V}, 0 \mathrm{~V}$, and $4 \mathrm{~V}$ are shown in Figure 2(a). Indeed, it is observed that the applied voltage leads to either enhancement or reduction of the pinning field, depending on the sign of the applied voltage. A negative voltage increases the pinning strength, as seen in the top row of images. On the other hand, a positive voltage leads to a significantly reduced pinning field $(<4.0 \mathrm{mT})$, as seen in the bottom row of images.

In Figure 2(b), we have performed a systematic measurement of the pinning field as a function of voltage. At each voltage, the experiment outlined above is repeated $N=10$ times and the average pinning field is plotted, where the error bar represents the standard deviation of the measurement (which is $\sqrt{N-1}$ times larger than the error in the average). It is found that the pinning strength changes linearly with the applied voltage. Comparing the red and blue lines in Figure 2(b), it is observed that the pinning strength is altered similarly for the two $H$-field polarities, which is compatible with the idea that we are tuning the DW pinning barrier and further excludes effects of the leakage current. The effect of the voltage on DW pinning (average absolute slope of Figure 2(b)) quantifies to $0.22 \pm 0.01 \mathrm{mT} / \mathrm{V}$ in the studied range. Somewhere beyond $4 \mathrm{~V}$, the device suffered from dielectric breakdown after which the junction was destroyed. Looking at the error bars, it can be seen that there is a certain spread among individual measurements of the pinning field at constant voltage. This can be attributed to thermally activated depinning: even if a field below the "true" (zero-temperature) pinning field is applied, there is a certain probability for the DW to overcome the energy barrier, ${ }^{28}$ leading to a statistical distribution in the measured pinning field.
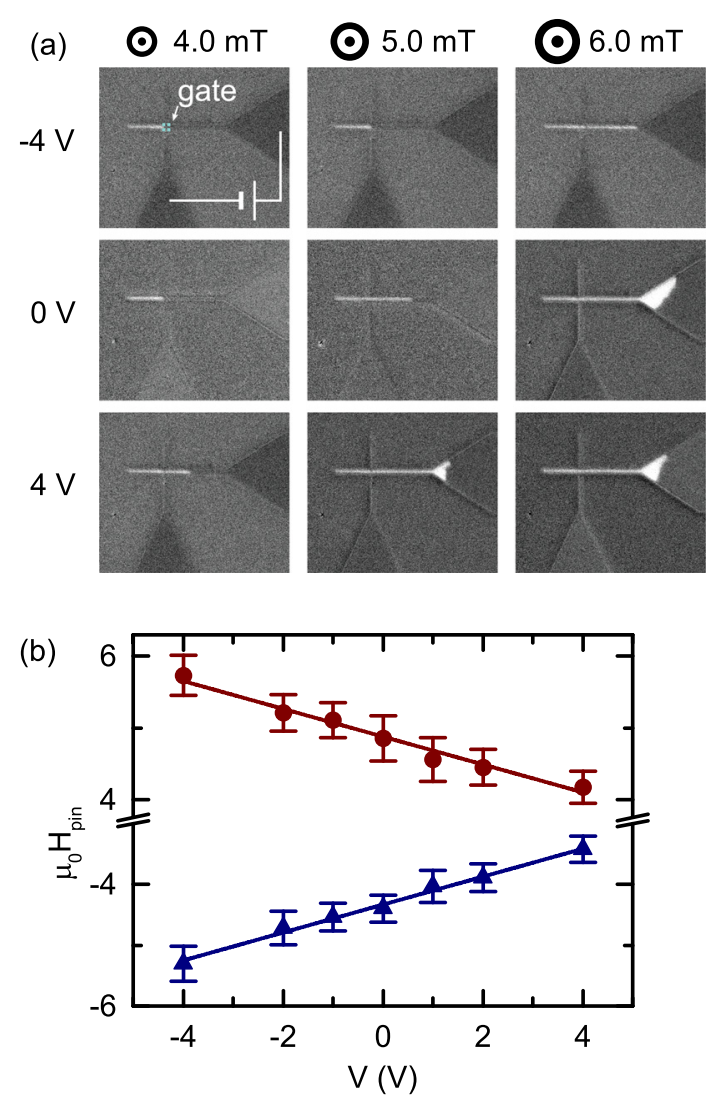

FIG. 2. (a) Kerr microscopy images of DW behavior around the gate as a function of applied magnetic field (horizontal) and applied voltage (vertical). It is seen that the DW moves past the gate at a field strength which depends on the voltage. (b) Systematic measurement of the pinning field as a function of voltage. Similar slopes are found for up-down DWs (red discs, $0.23 \pm 0.01 \mathrm{mT} / \mathrm{V}$ ) and down-up DWs (blue triangles, $0.19 \pm 0.01 \mathrm{mT} / \mathrm{V}$ ). The error bar represents the standard deviation of the measurement (10 repeats). 
We now turn to a simple DW-energy model in order to verify these results, in particular, the magnitude of the observed pinning effect. In the previous experiments on permanent DW pinning sites created by Focused Ion Beam irradiation, ${ }^{29,30}$ we developed a model to describe the pinning field at an anisotropy step $\Delta K$ (Ref. 30)

$$
H_{\text {pin }}=\frac{\Delta K}{2 \mu_{0} M_{\mathrm{s}}} \times \frac{2 \lambda}{\delta} \tanh \frac{\delta}{2 \lambda} .
$$

Here, $M_{\mathrm{s}}$ is the saturation magnetization $(1400 \mathrm{kA} / \mathrm{m}$ for Co), and $\lambda$ is the width of the DW. We estimate $\lambda=\sqrt{A / K}=7.7 \mathrm{~nm}$, assuming the exchange stiffness $A=16 \mathrm{pJ} / \mathrm{m}$ (Ref. 35) and the effective uniaxial anisotropy $K=0.27 \mathrm{MJ} / \mathrm{m}^{3}$, measured by SQUID magnetometry. Furthermore, two important tunable parameters can be identified: (1) the magnitude of the step in the anisotropy $\Delta K$, and (2) the length scale $\delta$ of the anisotropy gradient, as defined in Figure 1(d).

To estimate the magnitude of $\Delta K$ in our experiments, we use results that were obtained on very large junctions made of the same material, where the DW velocity was altered using an electric field. ${ }^{21}$ In this experiment, it was found that in order to explain the observed changes in DW velocity, the change of the $\mathrm{Co} / \mathrm{AlO}_{x}$ surface magnetic anisotropy per applied electric field amounted to $14 \pm 2 \mathrm{fJ} \mathrm{V}^{-1}$ $\mathrm{m}^{-1}$. Multiplying this value by the electric field $V / t$ and dividing by the Co thickness $(0.8 \mathrm{~nm})$ yields the effective (volume) anisotropy change $\Delta K$. Less straightforward is the estimation of the length scale of the anisotropy gradient, $\delta$. The electron density at the $\mathrm{Co} / \mathrm{AlO}_{x}$ interface, which determines $K$, is not expected to jump instantaneously at $x=0$, where the top electrode starts. Rather, the electron density is expected to change gradually, and the characteristic length $\delta$ of this change might depend on the thickness $t$ of the insulating layer and its relative permittivity $\epsilon_{r}$. To estimate this, a 2D COMSOL model is developed which calculates the electric field distribution in the layer system around the gate region. Taking $\epsilon_{r}=7$ for $\mathrm{AlO}_{x}$ and $t=10 \mathrm{~nm}$, we find the voltage distribution as sketched in Figure 3(a). It is expected that the PMA scales with the electric field at the $\mathrm{Co} / \mathrm{AlO}_{x}$ interface (dashed line), which is plotted as a function of $x$ in Figure 3(b) for various $t$. As expected, it is observed that $\delta$ increases with $t$, which is visualized by the shaded areas in Figure 3(b). We define the gradient length $\delta$ by extrapolating the slope at the center of the profile to the full profile height. If we incorporate the values of $\delta$ extracted from Figure 3(b) into the model of Eq. (1), we get the pinning strength as a function of voltage shown in Figure 3(c) for varying $\mathrm{AlO}_{x}$ thickness $t$. The slope of the voltage induced pinning field for a $10 \mathrm{~nm}$ thick $\mathrm{AlO}_{x}$ layer is $0.44 \mathrm{mT} / \mathrm{V}$ (red line in Figure $3(\mathrm{c})$ ), of the same order as the value $0.22 \pm 0.01 \mathrm{mT} / \mathrm{V}$ found in the experiment (dashed orange line in Figure 3(c)). We therefore think this model captures the essential physics of the observed effect, although fully quantitative agreement cannot be claimed due to various subtleties, such as the uncertainty in the voltage dependence of $K$, the 1D-model simplification, the roughness of the top electrode, the precise value of the DW width $\lambda$, and the fact that DW pinning is a

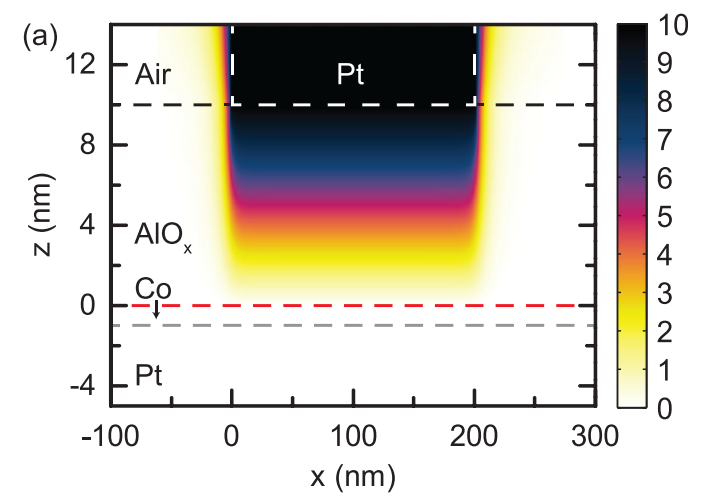

(b)
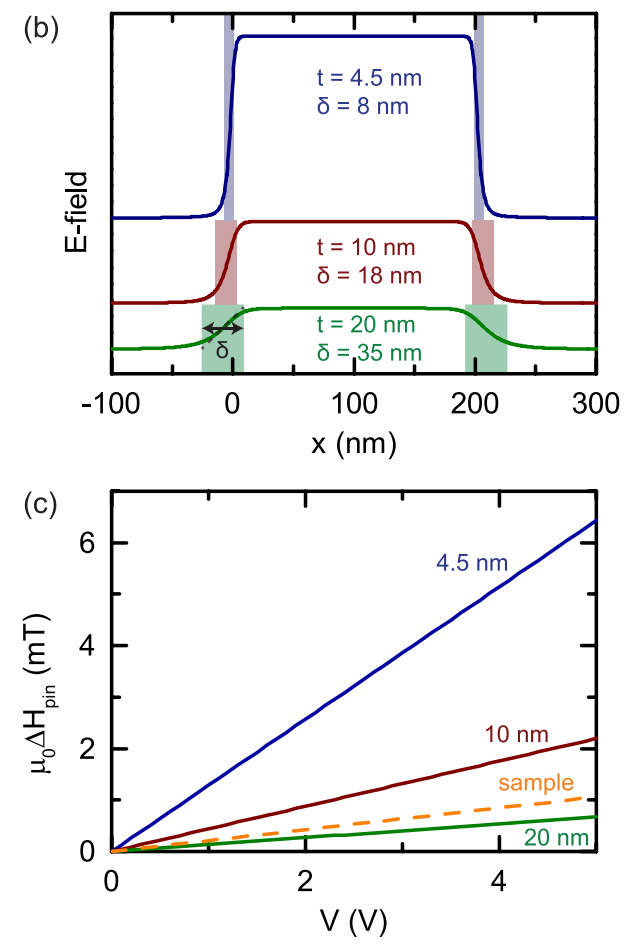

FIG. 3. (a) Calculation of the voltage profile at the gate for an $\mathrm{AlO}_{x}$ thickness $t=10 \mathrm{~nm}$. The resulting E-field as a function of $x$ at the $\mathrm{Co} / \mathrm{AlO}_{x}$ interface (red dashed line) is plotted in (b) for $t=4.5 \mathrm{~nm}$ (blue), $t=10 \mathrm{~nm}$ (red), and $t=20 \mathrm{~nm}$ (green), where the shaded area indicates the width of the Efield profile, which is seen to increase for thicker $t$. Panel (c) shows the expected DW pinning strength as a function of voltage based on the profile width $\delta$ plugged into Eq. (1), along with a fit to the experimental data (dashed orange line).

thermally assisted process. We should also note that in the model, pinning is absent at $0 \mathrm{~V}$, whereas in the experiment the strength of an already existing pinning barrier is altered, although this does not have major consequences for the physics involved.

In conclusion, we have demonstrated a pinning site in a $\mathrm{Pt} / \mathrm{Co} / \mathrm{AlO}_{x} \mathrm{DW}$ conduit which can be tuned by applying an electric field. The pinning field was shown to change linearly as a function of applied voltage at $0.22 \pm 0.01 \mathrm{mT} / \mathrm{V}$. A simple micromagnetic $1 \mathrm{D}$ model in combination with calculations of the electric field distribution yielded a reasonable reproduction of the observed effect. The model suggests that not only the magnitude of the electric field but also the width of the electric field distribution at the gate edge is important in engineering an efficient pinning site. Although a much stronger pinning field was achieved by Bauer et al. ${ }^{26}$ using 
the effect of electromigration, our result is caused by the completely different mechanism of tuning the electron density at the interface which might have its own advantages, such as a potentially higher toggling speed. In a preliminary experiment, we have observed that a pinned DW depins relatively quickly $(\sim 1 \mathrm{~s})$ after the voltage is removed. It is therefore worthwhile to investigate the speed limit of this mechanism for application in future devices.

This work is part of the research programme of the Foundation for Fundamental Research on Matter (FOM), which is part of the Netherlands Organisation for Scientific Research (NWO). This work was supported by NanoNextNL, a Micro and Nanotechnology Consortium of the Government of the Netherlands and 130 partners.

${ }^{1}$ M. K. Niranjan, C.-G. Duan, S. S. Jaswal, and E. Y. Tsymbal, Appl. Phys. Lett. 96, 222504 (2010)

${ }^{2}$ F. Bonell, S. Murakami, Y. Shiota, T. Nozaki, T. Shinjo, and Y. Suzuki, Appl. Phys. Lett. 98, 232510 (2011).

${ }^{3}$ Y. Shiota, S. Murakami, F. Bonell, T. Nozaki, T. Shinjo, and Y. Suzuki, Appl. Phys. Express 4, 043005 (2011).

${ }^{4}$ M. Endo, S. Kanai, S. Ikeda, F. Matsukura, and H. Ohno, Appl. Phys. Lett. 96, 212503 (2010).

${ }^{5}$ T. Maruyama, Y. Shiota, T. Nozaki, K. Ohta, N. Toda, M. Mizuguchi, A. A. Tulapurkar, T. Shinjo, M. Shiraishi, S. Mizukami, Y. Ando, and Y. Suzuki, Nat. Nanotechnol. 4, 158 (2009).

${ }^{6}$ T. Nozaki, Y. Shiota, M. Shiraishi, T. Shinjo, and Y. Suzuki, Appl. Phys. Lett. 96, 022506 (2010).

${ }^{7}$ S.-S. Ha, N.-H. Kim, S. Lee, C.-Y. You, Y. Shiota, T. Maruyama, T. Nozaki, and Y. Suzuki, Appl. Phys. Lett. 96, 142512 (2010).

${ }^{8}$ M. Weisheit, S. Fähler, A. Marty, Y. Souche, C. Poinsignon, and D. Givord, Science 315, 349 (2007).

${ }^{9}$ C. Fowley, K. Rode, K. Oguz, H. Kurt, and J. M. D. Coey, J. Phys. D: Appl. Phys. 44, 305001 (2011).

${ }^{10}$ T. Seki, M. Kohda, J. Nitta, and K. Takanashi, Appl. Phys. Lett. 98, 212505 (2011).

${ }^{11}$ H. Ohno, D. Chiba, F. Matsukura, T. Omiya, E. Abe, T. Dietl, Y. Ohno, and K. Ohtani, Nature 408, 944 (2000).

${ }^{12}$ M. Zhernenkov, M. R. Fitzsimmons, J. Chlistunoff, J. Majewski, I. Tudosa, and E. E. Fullerton, Phys. Rev. B 82, 024420 (2010).

${ }^{13}$ D. Chiba, S. Fukami, K. Shimamura, N. Ishiwata, K. Kobayashi, and T. Ono, Nature Mater. 10, 853 (2011).
${ }^{14}$ Y. Shiota, T. Nozaki, F. Bonell, S. Murakami, T. Shinjo, and Y. Suzuki, Nature Mater. 11, 39 (2011).

${ }^{15}$ W.-G. Wang, M. Li, S. Hageman, and C. L. Chien, Nature Mater. 11, 64 (2011).

${ }^{16}$ L. Liu, C.-F. Pai, D. C. Ralph, and R. A. Buhrman, e-print arXiv:1209.0962.

${ }^{17}$ J. Dean, M. T. Bryan, T. Schrefl, and D. A. Allwood, J. Appl. Phys. 109, 023915 (2011).

${ }^{18}$ T.-K. Chung, G. P. Carman, and K. P. Mohanchandra, Appl. Phys. Lett. 92, 112509 (2008).

${ }^{19}$ T. H. E. Lahtinen, K. J. A. Franke, and S. van Dijken, Sci. Rep. 2, 258 (2012).

${ }^{20}$ D. Chiba, M. Kawaguchi, S. Fukami, N. Ishiwata, K. Shimamura, K. Kobayashi, and T. Ono, Nature Commun. 3, 888 (2012).

${ }^{21}$ A. J. Schellekens, A. van den Brink, J. H. Franken, H. J. M. Swagten, and B. Koopmans, Nature Commun. 3, 847 (2012).

${ }^{22}$ U. Bauer, S. Emori, and G. S. D. Beach, Appl. Phys. Lett. 101, 172403 (2012).

${ }^{23}$ U. Bauer, S. Emori, and G. S. D. Beach, Appl. Phys. Lett. 100, 192408 (2012).

${ }^{24}$ A. Bernand-Mantel, L. Herrera-Diez, L. Ranno, S. Pizzini, J. Vogel, D. Givord, S. Auffret, O. Boulle, I. M. Miron, and G. Gaudin, Appl. Phys. Lett. 102, 122406 (2013).

${ }^{25}$ U. Bauer, M. Przybylski, J. Kirschner, and G. S. D. Beach, Nano Lett. 12, 1437 (2012)

${ }^{26}$ U. Bauer, S. Emori, and G. S. D. Beach, Nat. Nanotechnol. 8, 411 (2013).

${ }^{27}$ S. S. P. Parkin, M. Hayashi, and L. Thomas, Science 320, 190 (2008).

${ }^{28}$ C. Burrowes, A. P. Mihai, D. Ravelosona, J. Kim, C. Chappert, L. Vila, A. Marty, Y. Samson, F. Garcia-Sanchez, L. D. Buda-Prejbeanu, I. Tudosa, E. E. Fullerton, and J.-P. Attané, Nat. Phys. 6, 17 (2010).

${ }^{29}$ J. H. Franken, M. Hoeijmakers, R. Lavrijsen, J. T. Kohlhepp, H. J. M. Swagten, B. Koopmans, E. van Veldhoven, and D. J. Maas, J. Appl. Phys. 109, $07 \mathrm{D} 504$ (2011)

${ }^{30}$ J. H. Franken, M. Hoeijmakers, R. Lavrijsen, and H. J. M. Swagten, J. Phys. Condens. Matter 24, 024216 (2012).

${ }^{31}$ J. H. Franken, H. J. M. Swagten, and B. Koopmans, Nat. Nanotechnol. 7, 499 (2012).

${ }^{32}$ I. M. Miron, T. Moore, H. Szambolics, L. D. Buda-Prejbeanu, S. Auffret, B. Rodmacq, S. Pizzini, J. Vogel, M. Bonfim, A. Schuhl, and G. Gaudin, Nature Mater. 10, 419 (2011).

${ }^{33}$ A. Manchon, C. Ducruet, L. Lombard, S. Auffret, B. Rodmacq, B. Dieny, S. Pizzini, J. Vogel, V. Uhlir, M. Hochstrasser, and G. Panaccione, J. Appl. Phys. 104, 043914 (2008).

${ }^{34}$ J. Kimling, T. Gerhardt, A. Kobs, A. Vogel, S. Wintz, M.-Y. Im, P. Fischer, H. Peter Oepen, U. Merkt, and G. Meier, J. Appl. Phys. 113, 163902 (2013).

${ }^{35}$ P. Metaxas, J. Jamet, A. Mougin, M. Cormier, J. Ferré, V. Baltz, B. Rodmacq, B. Dieny, and R. Stamps, Phys. Rev. Lett. 99, 217208 (2007). 